\title{
Temperature-dependent refractive index measurements of wafer-shaped InAs and InSb
}

\author{
Glen D. Gillen, ${ }^{1, *}$ Chris DiRocco, ${ }^{2}$ Peter Powers, ${ }^{2}$ and Shekhar Guha ${ }^{3}$ \\ ${ }^{1}$ Department of Physics, California Polytechnic State University, San Luis Obispo, California 93407, USA \\ Electro-Optics Program, University of Dayton, Dayton, Ohio 45469, USA \\ ${ }^{3}$ Materials and Manufacturing Directorate, Air Force Research Laboratories, Wright-Patterson Air Force Base, Ohio 45433, USA
}

*Corresponding author: ggillen@calpoly.edu

Received 14 August 2007; accepted 16 November 2007;

posted 28 November 2007 (Doc. ID 86461); published 7 January 2008

\begin{abstract}
An experimental method is introduced to measure the refractive index and its temperature dependence for wafer-shaped infrared materials over a continuous temperature range. Using a combination of Michelson interferometry, Fabry-Perot interferometry, and a temperature-controlled cryostat in a laser micrometer, refractive index values and their temperature coefficients can be measured for any specific temperature within a desired temperature range. Measurements are reported for InAs and InSb for a laser wavelength of $10.59 \mu \mathrm{m}$. (C) 2008 Optical Society of America
\end{abstract}

OCIS codes: $120.3180,120.4290,160.4760,160.6000$.

\section{Introduction}

The refractive index, $n$, is a fundamental parameter of any optical material. The linear component of the refractive index of a material is responsible for both the propagation speed (real part) and the absorption (imaginary part) of light waves within the material. For boundaries between different optical media, refraction of the light wave vector, and transmission and reflection coefficients are a result of the optical impedance mismatch between the two different linear refractive indices. The nonlinear component of the refractive index of an optical material is responsible for many optically induced phenomena including self-focusing, self-defocusing, self-phase modulation, etc. All components of a material's refractive index are temperature-dependent. Many visible light and infrared optical components and systems are subject to extreme changes in temperature (from liquid He detectors to above-room-temperature nonlinear optical crystals). Accurate knowledge of the linear refractive index and the temperature coefficient of the refractive index (TCRI), $(1 / n)(\mathrm{d} n / \mathrm{d} T)$, is required for accurate interpretation and analysis of

0003-6935/08/020164-05\$15.00/0

(C) 2008 Optical Society of America the performance of existing optical systems, in addition to theoretically modeling and predicting the performance of specific linear and nonlinear optical components and systems subject to thermal fluctuations.

Historical methods used to measure $n$ and the TCRI include minimum deviation [1,2], ellipsometry $[3,4]$, and immersion [5,6]. Mid- and long-wave infrared materials present a unique challenge for experimentalists to measure the refractive index and its temperature coefficient. Many infrared materials are typically grown as thin wafers having flat and parallel surfaces, and have high refractive indices. Thus the commonly used (and highly accurate) minimum deviation method is ruled out for nondestructive and sample-specific testing due to the physical shape and thickness limitations of these materials. The method of ellipsometry is effective if surface properties are desired, but is not ideal for bulk measurements. The method of immersion is also ruled out as thermal immersion oils with high refractive indices do not exist. Recently, interferometric methods have been used to perform sample-specific bulk refractive index measurements on wafer-shaped materials $[7,8]$, but results were limited to only two temperatures: room temperature and cryogenic. 
In this paper we demonstrate how an angledependent Michelson-Fabry-Perot interferometer, a temperature-dependent Fabry-Perot interferometer, and a temperature-dependent optical micrometer can be used to accurately measure the real part of the temperature-dependent index of refraction of wafershaped optical materials. Using this method, measurements can be conducted over a continuous temperature range, and without any prior knowledge of the material's optical and thermal properties.

The experimental methods presented in this paper are applied to the materials of InAs and InSb. To the best of the authors' knowledge, the temperature coefficient of the refractive index of InAs and InSb have only been recorded or theorized for room temperature $[9,10]$. We report here experimentally measured temperature-dependent values of the refractive index and its temperature coefficients for InAs and $\mathrm{InSb}$ over a continuous temperature range.

\section{Method}

The procedure presented here is an extension of our previous method [7,8] employing Michelson and Fabry-Perot interferometry for refractive index measurements at two temperatures: room temperature and cryogenic. Here we include the use of a temperature-dependent Fabry-Perot interferometer and a temperature-controlled cryostat in a laser micrometer to measure refractive index values over a continuous temperature range. Detailed discussion of decoupling the refractive index from the material thickness and measuring the refractive index of materials at room and cryogenic materials is presented in $[7,8]$, but will briefly be summarized here for completeness. The general experimental setup for all of the interferometric experiments is illustrated in Fig. 1.

If the flipper mirror is positioned so that the beam passes by it, the laser is incident into the Michelson interferometer at the top of Fig. 1. The vacuum

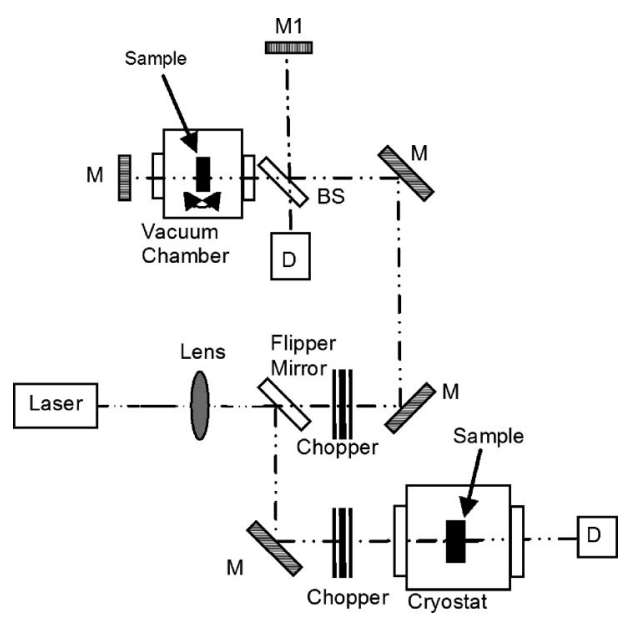

Fig. 1. Schematic of the angle-dependent Michelson interferometry setup (top) and the temperature-dependent Fabry-Perot interferometry setup (bottom). chamber incorporates a rotation feedthrough so that the wafer-shaped material can be rotated, varying the angle of incidence between the laser beam and the sample surface. The optical path through the sample arm of the Michelson interferometer is dependent upon the sample orientation with respect to the incident beam. The phase difference between the sample arm of the interferometer and the reference arm of the interferometer becomes [7]

$$
\phi_{m}(\theta)=\frac{4 \pi L}{\lambda}\left(\sqrt{n^{2}-\sin ^{2} \theta}+1-\cos \theta\right)
$$

where $L$ is the material thickness, $\lambda$ is the laser wavelength in vacuum, $n$ is the real part of the linear refractive index, and $\theta$ is the angle of incidence. The observed output intensity of the Michelson interferometer will oscillate due to the interference of the angle-dependent phase of the sample arm and the constant phase of the reference arm. If the thickness of the material is known, the absolute refractive index can be extracted from the intensity modulation data using the angle-dependent phase relationship in Eq. (1) for the material's temperature during the interferometric scan.

The Michelson interferometer of Fig. 1 can be transformed into a double-pass Fabry-Perot interferometer by blocking the reference arm beam between the beam splitter, BS, and the reference mirror, M1. In this arrangement, the material itself acts as an etalon due to the flat and parallel surfaces of the material. Multiple internal reflections within the sample interfere with one another due to the optical path difference between subsequent internal reflection paths. The phase difference between subsequent internal reflections can be expressed as [8]

$$
\phi_{f}(\theta)=\frac{4 \pi L}{\lambda} \sqrt{n^{2}-\sin ^{2} \theta}
$$

As with the Michelson interferometer, the output intensity of the double-pass Fabry-Perot interferometer will oscilate due to the angle-dependent phase expressed in Eq. (2). If the thickness of the material is known, then the refractive index can be determined by fitting the observed angle-dependent intensity using the angle-dependent phase relationship of Eq. (2). The vacuum chamber of Fig. 1 is designed such that interferometric scans can be performed at either room temperature or at cryogenic temperatures.

Measuring the refractive index using either Michelson or Fabry-Perot interferometry requires prior accurate knowledge of the material's thickness and thermal expansion properties at the temperature under which the scan is performed. However, if the angle-dependent phase information for both techniques is recorded, the temperaturedependent refractive index can be decoupled from the temperature-dependent material thickness by 
comparing the angle-dependent phase difference between the two interferometric methods [8]. The angle-dependent phase difference is simply

$$
\phi_{m}(\theta)-\phi_{f}(\theta)=\frac{4 \pi L}{\lambda}(1-\cos \theta)
$$

and is dependent only upon the material thickness. Using Eq. (3), the material thickness can be determined for the current material temperature using the measured phase differences as a function of $\theta$. Once the material thickness is determined, data from either interferometric method can be used to determine the absolute refractive index for the current temperature.

Using the phase relationships of Eqs. (1)-(3), independent and absolute measurements can be obtained for the material thickness and refractive index for any temperature where the material's temperature is stable and constant. Currently available commercial temperature-controlled dewars do not allow for precise rotation of the sample at a fixed temperature. Previously, we have employed a home-built dewar system with a precision rotation feed through $[7,8]$. The primary limitation of the setup is that measurements can only be performed at room temperature and the base cryogenic temperature. Here we extend the method to obtain a continuous measurement of the refractive index between the two temperature extremes. Once the continuous temperature dependence of $n$ is measured the TCRI can be determined over the same experimental temperature range.

By inserting the flipper mirror of Fig. 1 into the beam path, the laser is diverted to a temperaturecontrolled cryostat system. Here the sample serves as an etalon in a single-pass Fabry-Perot interferome-

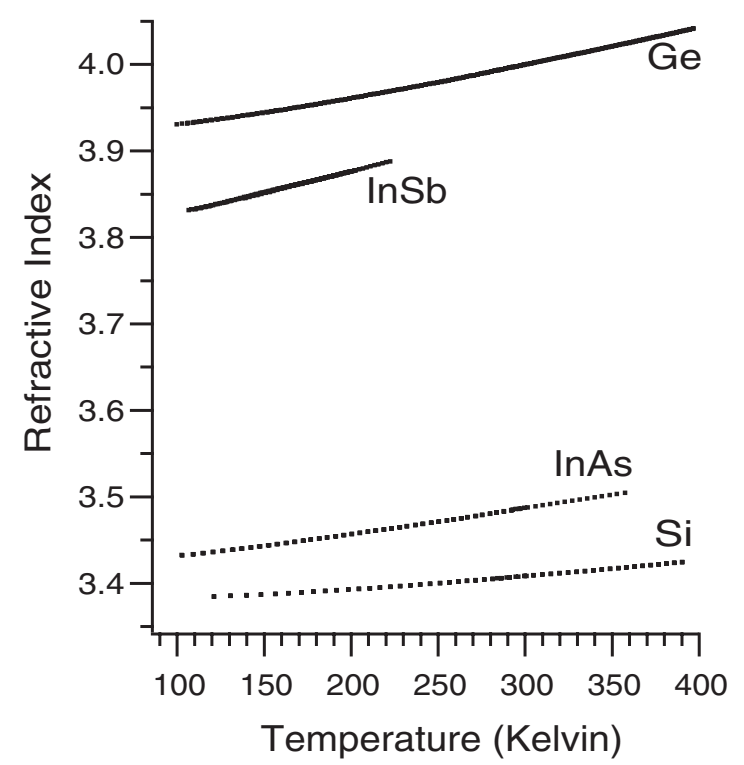

Fig. 2. Temperature-dependent absolute refractive index for germanium and silicon (calibration materials), as well as InAs and InSb.

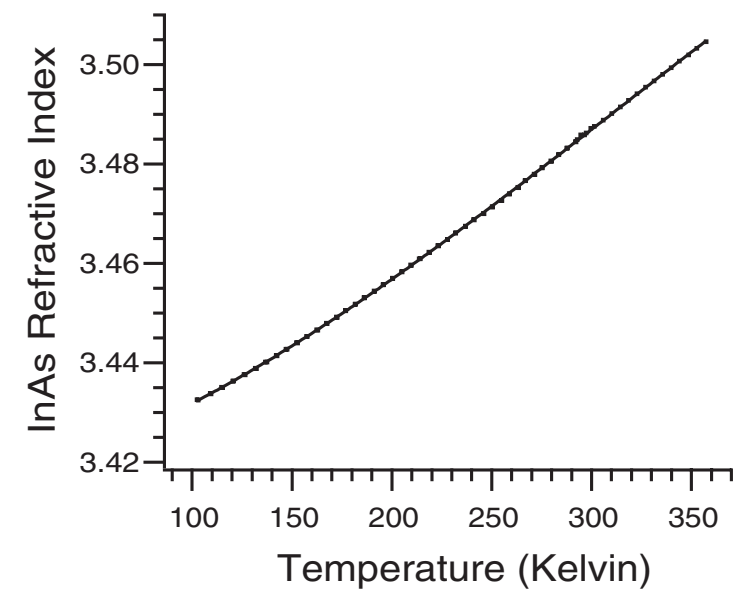

Fig. 3. Temperature-dependent absolute refractive index for InAs from 102 to $357 \mathrm{~K}$.

ter. By orienting the material so that the beam is normally incident and varying the temperature of the material the phase between subsequent internal reflections becomes temperature-dependent and follows

$$
\phi_{f}(T)=\frac{4 \pi n(T) L(T)}{\lambda}
$$

As the temperature of the material is varied, the transmitted light intensity will modulate due to the temperature-dependent phase.

A custom sample mount was designed for the cryostat system such that the material wafer is held horizontally and supported only from underneath allowing the sample to freely expand or contract due to temperature changes. The cryostat system is then placed in the beam path of a laser micrometer, and the material's thickness as a function of temperature, $L(T)$, is recorded. The laser micrometer is composed of a $\mathrm{HeNe}$ laser beam that is scanned in a vertical

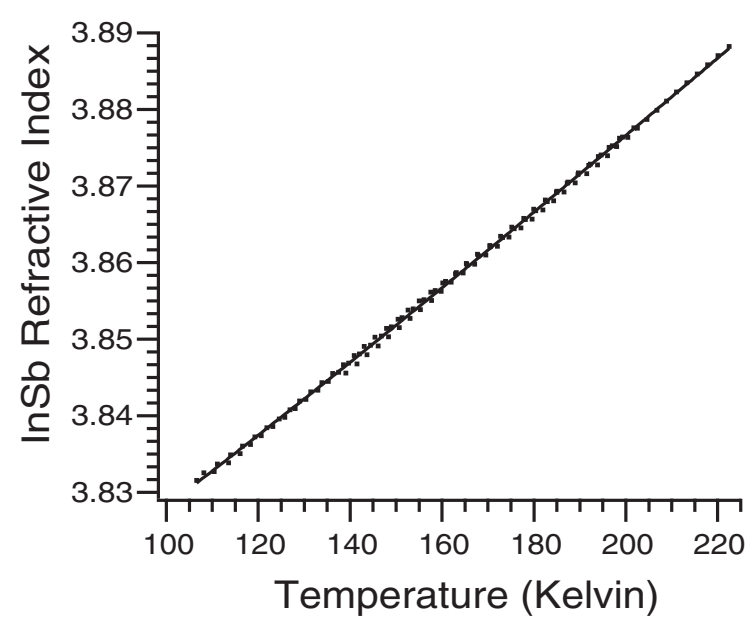

Fig. 4. Temperature-dependent absolute refractive index for InSb from 104 to $222 \mathrm{~K}$. 
Table 1. Polynomial Fits to the Measured Temperature-Dependent Refractive Indices of Ge, Si, InAs, and InSb, Where the Temperature is in Kelvins

$n(T)=A+B T+C T^{2}+D T^{3}$

\begin{tabular}{ccrrr}
\hline $\begin{array}{c}\text { Material } \\
\text { (Temperature Range) }\end{array}$ & \multicolumn{1}{c}{$A$} & $B \times 10^{-5}$ & $C \times 10^{-7}$ & $D \times 10^{-10}$ \\
\hline Ge (99.6-397 K) & $3.9135 \pm 0.0003$ & $9.21 \pm 0.41$ & $8.78 \pm 0.17$ & $-7.48 \pm 0.23$ \\
Si (121-390 K) & $3.3825 \pm 0.0003$ & $-5.01 \pm 0.37$ & $6.24 \pm 0.15$ & $-5.67 \pm 0.19$ \\
InAs (102-357 K) & $3.4165 \pm 0.0005$ & $9.05 \pm 0.74$ & $7.05 \pm 0.34$ & $-7.46 \pm 0.49$ \\
InSb (106-222 K) & $3.7869 \pm 0.0008$ & $36.13 \pm 0.77$ & $6.0 \pm 1.7$ & $-8.16 \pm 0.93$ \\
\hline
\end{tabular}

plane. The thickness of an object placed in that plane is measured according to the time the HeNe beam is blocked by the material; essentially transforming a thickness measurement into a time measurement. Application of the measured temperature-dependent thickness, $L(T)$, to the temperature-dependent phase modulation, $\phi_{f}(T)$, yields a temperature-dependent curve of the refractive index, $n(T)$, between the two endpoint values measured using the MichelsonFabry-Perot method previously described.

\section{Results and Discussion}

The laser used is a $200 \mathrm{~mW}$ tunable wavelength $\mathrm{CO}_{2}$ laser tuned to a wavelength of $10.591 \mu \mathrm{m}$. For interferometric measurements the laser beam is chopped to establish a master frequency for the experimental system. The orientation angle of the material inside of the vacuum chamber is controlled using a precision rotation stage, general-purpose interface bus (GPIB) motion controller, and a rotation feedthrough vacuum port. For the single-pass temperature-dependent Fabry-Perot interferometer the temperature is controlled and recorded using a temperature controller interfaced to a computer. The signal from the infrared detectors for either setup is recorded using a lock-in amplifier and the chopper's master frequency.

Measurements were conducted for Ge and Si to test the method and the system's accuracy. Once the analysis of the data collected for Ge and Si was complete, material samples of InAs and InSb were studied. Using the method outlined in Section 2 , the refractive index as a function of temperature over a specific temperature range is obtained. Temperature-dependent refractive indices for all materials are illustrated in Fig. 2. Figures 3 and 4 are expanded views of the results for InAs and InSb, respectively. The resulting refractive index versus temperature data points are mathematically modeled over the given temperature range using a third-order polynomial fit. The coefficients of each fit for each material are reported in Table 1, along with the measured temperature range for each material. Refractive index measurements obtained for $\mathrm{Ge}$ and $\mathrm{Si}$ agree with previously reported values at $300 \mathrm{~K}$ [11]. The measured value of InAs at $300 \mathrm{~K}$ is 3.49 as compared to a previously published value of 3.51 for a wavelength of $10.6 \mu \mathrm{m}$ [12].

For the InSb sample studied, the light absorption was less than $10 \%$ for all temperatures below $175 \mathrm{~K}$. Above $175 \mathrm{~K}$, the absorption of light due to thermally induced charge carrier production and increasing values of the imaginary part of the refractive index occurs. Most temperature scans of InSb were conducted up to a temperature of $\sim 220 \mathrm{~K}$ where the absorption rate reached $31 \%$. For higher temperatures, the charge carrier production continues to increase leading to an absorption rate of $50 \%$ by $240 \mathrm{~K}$. Extrapolating the mathematical fit for InSb of Table 1 (for comparison purposes) a real refractive index value of 3.93 at $300 \mathrm{~K}$ is calculated; which agrees well with the accepted value of 3.94 at $300 \mathrm{~K}$ for $10.6 \mu \mathrm{m}$ light [12].

The temperature coefficient of the refractive index of each material studied is reported in Table 2. The coefficients for each material in Table 2 are obtained from the mathematical fits of Table 1 to the measured data for the temperature-dependent refractive index and a recalculation of the $(1 / n)(\mathrm{d} n / \mathrm{d} T)$ expression to a third-order polynomial. For comparison with temperature-dependent calculations of the refractive index of semiconductors performed by Ghosh [13], Table 3 reports values calculated for a $(2 n)(\mathrm{d} n / \mathrm{d} T)$ function using the mathematical relationships found

Table 3. Comparison of $2 n(\mathrm{~d} n / \mathrm{d} T)$ Values Reported Here and Values Predicted by Ghosh [13] for $\mathrm{Ge}, \mathrm{Si}$, and InAs at a Temperature of $300 \mathrm{~K}$ easured Refractive Index Measurements over a Continuou Temperature Range, Where the Temperature is in Kelvins

\begin{tabular}{ccccc}
\hline \multicolumn{5}{c}{$\frac{1}{n} \frac{\mathrm{d} n(T)}{\mathrm{d} T}=A+B T+C T^{2}+D T^{3}$} \\
\\
\hline Material & $A \times 10^{-5}$ & $B \times 10^{-7}$ & $C \times 10^{-10}$ & $D \times 10^{-14}$ \\
\hline Ge & 2.33 & 4.53 & -6.30 & 6.05 \\
Si & -1.50 & 3.73 & -5.25 & 2.34 \\
InAs & 2.63 & 4.16 & -7.04 & 6.63 \\
InSb & 9.54 & 3.09 & -7.03 & 10.5 \\
\hline
\end{tabular}

\begin{tabular}{ccc}
\hline \multicolumn{3}{c}{$2 n \frac{\mathrm{d} n}{\mathrm{~d} T}\left(\times 10^{-3} / \mathrm{K}\right)$} \\
\hline Material & Using Tables & \\
\hline Ge & 1 and 2 & Predicted [13] \\
Si & 3.33 & 3.24 \\
InAs & 1.16 & 1.08 \\
\hline
\end{tabular}


in Tables 1 and 2. All values found in Table 3 are for a temperature of $300 \mathrm{~K}$.

\section{Conclusions}

We have extended our previous method for the nondestructive measurement of the refractive index of wafer-shaped infrared materials to yield temperature-dependent refractive index measurements over a continuous temperature range and calculation of the temperature-dependent coefficient of the refractive index for the material. Using this method we report temperature-dependent refractive indices and their temperature coefficient functions for InAs and InSb over the temperature ranges of 102 to $357 \mathrm{~K}$ and 106 to $222 \mathrm{~K}$, respectively.

\section{References}

1. R. N. Singh, D. P. Juyal, J. S. Bharguva, K. M. Mathur, O. S. Nagar, and A. N. Bhattacharyya, "An accurate visual method for refractive index measurement in near infrared," J. Phys. E 4, 2054-2058 (2004).

2. D. E. Zelmon, E. A. Hanning, and P. G. Schunemann, "Refractive-index measurements and Sellmeier coefficients for zinc germanium phosphide from 2 to $9 \mu \mathrm{m}$ with implications for phase matching in optical frequency-conversion devices," J. Opt. Soc. Am. B 18, 1307-1310 (2001).

3. D. E. Aspnes and A. A. Studna, "Dielectric functions and optical parameters of $\mathrm{Si}, \mathrm{Ge}, \mathrm{GaP}, \mathrm{GaAs}, \mathrm{GaSb}, \mathrm{InP}, \mathrm{InAs}$, and InSb from 1.5 to 6.0 eV," Phys. Rev. B 27, 985-1009 (1983).
4. Z. Huang and J. Chu, "The refractive index dispersion of $\mathrm{Hg}_{1-x} \mathrm{Cd}_{x} \mathrm{Te}$ by infrared spectroscopic ellipsometry," Infrared Phys. 42, 77-80 (2001).

5. S. Su, "A rapid and accurate procedure for the determination of refractive indices of regulated asbestos minerals," Am. Mineral. 88, 1979-1982 (2003).

6. M. Deetlefs, K. R. Sedden, and M. Shara, "Neoteric optical media for refractive index determination of gems and minerals," New J. Chem. 30, 317-326 (2006).

7. G. D. Gillen and S. Guha, "Refractive-index measurements of zinc germanium diphosphide at $300 \mathrm{~K}$ and $77 \mathrm{~K}$ by use of a modified Michelson interferometer," Appl. Opt. 43, 2054-2058 (2004).

8. G. D. Gillen and S. Guha, "Use of Michelson and Fabry-Perot interferometry for independent determination of the refractive index and physical thickness of wafers," Appl. Opt. 44, 344347 (2005).

9. P. Yu and M. Cardona, "Temperature coefficient of the refractive index of diamond- and zinc-blende-type semiconductors," Phys. Rev. B 2, 3193-3197 (1970).

10. P. J. L. Hervé and L. K. J. Vandamme, "Emperical temperature dependence of the refractive index of semiconductors," J. Appl. Phys. 77, 5476-5477 (1995).

11. H. W. Icenogle, B. C. Platt, and W. L. Wolfe, "Refractive indexes and temperature coefficients of germanium and silicon," Appl. Opt. 15, 2348-2351 (1976).

12. M. Levinshtein, S. Rumyantsev, and M. Shur, Handbook Series on Semiconductor Parameters (World Scientific, 1996), Vol. 1.

13. G. Ghosh, "Temperature dispersion of refractive indices in semiconductors,” J. Appl. Phys. 79, 9388-9389 (1996). 